\title{
BOUNDARY ELEMENT ANALYSIS OF TRANSMISSION LINES FOR TRANSVERSE ELECTROMAGNETIC WAVES
}

\author{
Dr. Hamdi A. El-Mikati*
}

\begin{abstract}
The boundary element method is used to compute the characteristic impedance and capacitance matrix of various types of transmission lines supporting transverse electromagnetic waves. Multiconductor lines between infinite ground planes are treated by combining the numerical solution with appropriate analytical representation of the potential in the conductor-free region. Comparison is made with available published results.
\end{abstract}

\section{INTRODUCTION}

Various types of transmission lines supporting transverse electromagnetic (TEM) waves have been developed and are widely used in high frequency communication systems. However, only few types with simple or highly symmetric structure do have exact formulae expressing the electric characteristics of the line in terms of its geometrical parameters. Considerable effort has therefore been spent on approximate and numerical techniques including, for example, approximate conformal mapping methods [1-6], variational methods [7], Fourier transiorm methods [8], finite differences [9], numerical methods based on Green's function integral equation approach [10-15], ...etc. A considerable body of literature has accumulated in the last few decades and a literature review may be found, for instance, in references $[1-15]$.

Recently, the boundary element method (BEM) has been introduced and efficiently applied to many potential problems involving Laplace's equation [16]. Beside its ability as a numerical method to deal with quite arbitrary geometric structures, it is of:en more memory-economic as compared with its rivat finite differences and finite elements methods. This advantage is due to the fact that the BEM confines the analysis of a homogeneous domain to the boundaries of the domain, and so the problem is in effect treated with one less dimension. Another advantage of the BEM is that, unlike other techniques based on integral equation formulation, the BEM does not require a knowledge of the Green's function of the particulat problem under consideration; the BEM formuiation involves onlv the Iree space (unbounded) Green's function, which for Laplace's equation is the well known logarithmic potential.

The present work has, therefore, been set to make use of the BEM as a basis for computer code $\{$ s suitable for use on a relatively small computer system and applicable to a wide class of TEM-tranmission lines. These include

1. Multiconductor lines in free space or inside a tubular conducting shield (boxed lines). The farniliar parallel-wires and coaxial lines are typical examples of this class.

2. Strip lines consisting of one or more strips arbitrarily located between two parallel ground planes.

3. Shielded microstrip lines (either boxed-line or a microstrip over a substrate of finite width between two infinite ground planes).

In all cases, the conductors are assumed to extend infinitely, parallel to the $z$ axis, but their cross-sections in the xy-plane can be of quite arbitrary shape.

* Department of Electronic Eng., Mansoura University 


\section{PARAMETERS OF MULTICONDUCTOR TEM-TRANSMISSION LINES}

This work is concerned with the computation of the distributed parameters (capacitance and inductance per unit length) as well as the characteristic admittance and coupling coefficients for TEM-transmission lines. For the sake of generality, we shall assume a multiconductor line. It is well known that the essential parameters of such a line are the elements of the Maxwellian capacitance matrix $C$, from which the characteristic admittances for the various operating TEM modes and the coupling coefticients can be easily obtained $[11-15]$. The capacitance matrix is detined as follows. Consider a system of n-conductors (plus ground) with potentials $V_{1}, V_{2}, \ldots, V_{n}$, and let $Q_{1}, Q_{2}, \ldots, Q_{n}$ be the corresponding total charges per unit length on the conductors. The potentials and charges are then related by

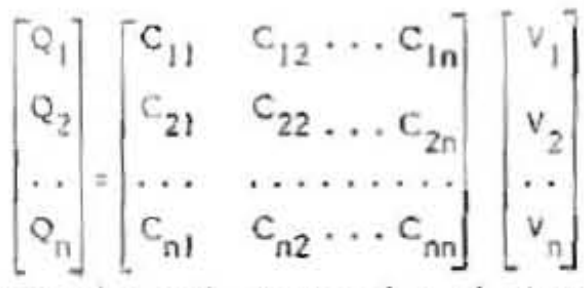

Reciprocity and conservation of charge imply the following known properties of the elements of the C-matrix $[14.15]$.

$$
\begin{aligned}
& c_{i j}=c_{i j} \\
& c_{i i}>0, \quad c_{i j}<0 \quad i \neq 1 \\
& \Sigma C_{i j} \geqslant 0
\end{aligned}
$$

The equality sign in the last equation holds for a 'closed system', where the summation runs over the grounded conductor. This is the case, lor instance, of a boxed line.

Similariy one can define the inductance matrix $\mathrm{L}$ which relates the fluxes and currents in the various conductors. It can be shown that. for a system of $n$ conductors in a homogeneous medium

$$
\mathrm{LC}=\mu \in \mathrm{I}
$$

where $\downarrow$ is the unit marrix of order $n$ and $u$ and $\epsilon$ are the permeability and permitivity of the medium, respectively.

The characteristic admittances are also readily obtained from $C$ [11]. If but a single conductor is involved $(n=1)$, the admittance is given by

$$
Y=\vee C \quad \ldots(4)
$$

where $v$ is the phase velocity of TEM-waves in the surrounding medium. In the case of a microstrip over a dielectric substrate

$$
Y=\sqrt{C_{d} / C_{0}} Y_{0}
$$

where $C_{d}$ is the capacitance per unit length of the actual microstrip structure and $C_{0}$ and $Y_{0}$ are the capacitance and characteristic admittance of the structure with air as dieiectric.

When two concuctors are involved $(n=2)$, there are two basic modes of operation: an odd mode $\left(V_{1}=1, V_{2}=-1\right)$, and an even one $\left(V_{1}=V_{2}=1\right)$. From (1), the corresponding total charges are

$$
\begin{aligned}
& Q_{\text {iodd }}=C_{i 1}-C_{i 2} \\
& Q_{\text {ieven }}=C_{i 1}+C_{i 2}
\end{aligned}
$$

The line admittances for these modes arc, therefore, 


$$
\begin{aligned}
& Y_{\text {iodd }}=v\left(C_{11}-C_{12}\right) / z \\
& Y_{\text {ieven }}=v\left(C_{14}+C_{12}\right)
\end{aligned}
$$

For $n>2$, the various line admittances for a partucular mode of operation may be obtained in a similar way.

\section{BOUNDARY ELEMENT ANALYSIS}

Following Collin $(17)$, the elements of the capacitance mutrix for a multiconductor line are obtained as follows. Let $u(x, y)$ be the potential function satisfyina the following boundary value problem

$$
\text { ane } \begin{array}{rlrl}
\nabla^{2} u_{1} & =0 & \text { outsice the conductor } \\
u_{j} & =1 & & \text { on the conductor } 1 \\
& =0 & \text { on sil other canductors }
\end{array}
$$

It then follows from (1) and Gauss's theorem that

$$
C_{11}, Q_{i}+-t f_{B,} q_{i} d c \quad i, i=1,2, \ldots+\text {, in }
$$

canducror where the integration is carried our along the
and $q j$ is the outward normal derivative of $u$.

The determination of the capacitance inatrix, therefore, requires finding in sotential functions of the type fiven by rquation (8). This repeated solution of the same potential problem tor different boundary conditions is considerably simplified in the boundary eitement method, which reduces the boundary value proolem to one of solving a system of linear equations with the source term being the linewri values of the potential and its normal derivative at the boundary nodes. If the system matrix is decomposed, for instance, using a Causuan elimination technique and stored in triangular form then, getring a new solurtion for a new set of bouncary values amounts ondy to a simple baci-suestitution precrst.

The BEM solves the boundary value problem $(8)$ as loilows. By means of the weighted residual method or Green's identity, the differential equation (X) Can be transformed into the following integration

$$
{ }_{p}+{ }_{B} \int_{\text {hu }} d c=\int_{B} \mathrm{gQ} d \mathrm{c}
$$

where $g$ is the free-space lunbourded Green's function and $h$ is the corresponding normal derivative. It is well known that $g=1 / 2 \pi$ in $(1 / r)$. The subscript $p$ in equation (10) means an arbitrary point inside a homogenecus region $R$ where $u$ satistius Laplace's equation and $B$ is the boundary of this region. In the special case when $p$ Lies on $B$, equation $(10)$ reduces to

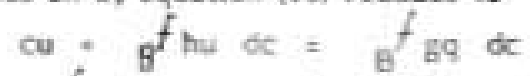

where $f$ denotes the Cauchy's principal value of the integral, $c=a / 2 \pi$, and a is the inner domain angle at $p$.

Equations $(10)$ and $(11)$ are the basic equations in the BEM anaiysis. The first of them is used to compute the fields at any internal point once the potential and its normal derivative are known on the boundary, These boundary values are in turn obtained by solving the boundacy integral equation (11). To this end, the boundary $B$ is discretized in the usual soparametric tinite element manner into, say, $N$-elements and the unknown functions $u$ and $q$ are approximated by suitable polynomials over each eiement. In the present work we confine ourselves to linear polynomials. Accocdingly, ecuation (it) is reduced to the discrete form

$$
c_{i} u_{i}+r h_{i j} u_{i}=t G_{i j} q_{i} \quad i=1,2, \ldots, N
$$

where $h_{1 j}$ and $B_{i j}$ are made up of inregrals over the two elements through node 


\section{E. 4 H. A. Elmikati}

$i$ of appropriate products of Green's functions and the interpolation polynomials. Detailed expressions for the various quantities and method of computation are given, for instance, in refernce [16]. In matrix form equation (12) becomes

$\mathrm{Hu}=\mathrm{G} \mathrm{q}$

where $u=\left(u_{1}, u_{2}, \ldots, u_{n}\right)^{\top}$ and $q=\left(q_{1}, q_{2}, \ldots, q_{n}\right)^{\top}$. Matrices $G$ and $H$ depend solely on the geometry of the region $R$, while vectors $u$ and $q$ comprise the values of the potential and its normal derivative at the boundary nodes of R. Some of these values are given from the boundary conditions or, in some cases, can be deduced from the symmetry of the problem if any. The remaining boundary values are obtained by solving the linear system (13). Next we consider some numerical examples with known analytical or numerical solution to compare with.

\section{NUMERICAL EXAMPLES}

\section{1- Soxed Lines and Open-wire Lines}

The BEM equations in this case are set for the region outside the conductors with the path of integration as shown in Fig. 1. In the case of a boxed-line, the outermost boundary $B_{0}$ coinicides with the inner surface of the shield. However, for a system of conductors in free-space, $B_{0}$ is taken to be a circle of infinitely large radjus. Assuming that the potential is regular (zero) at infinity, the contribution of $\mathrm{B}_{0}$ to the line integral vanishes in the latter case and the path of integration is confined to the conducting boundaries.

Table 1-3 present the capacitance of a coaxial system utilizing conductors of both circular and square cross-sections. The BEM results are compared with those obtained by Lin using conformal mapping techniques [4]. In case 1 these techniques give an exact result, while in cases 2 and 3 they give oniy upper and fower limits for the characteristic impedance. The BEM values lie within these limits and satisfactory agree with the exact values in most cases. Also the BEM has been used to compute the characteristic impedance when one of the conductors (or both) is rectangular with its diagonals tilted at an arbitrary angle with respect to the other conductor. Such results are useful, for example, when the structure is used as a coaxial reflection standard [10].

Table 4 gives the capacitance matrix and the odd-mode characteristic impedance of a shieided two-wire line. The accuracy of the BEM results are rather satisfactory as may be seen by checking the properties of the capacitance coefficients (equation (2) ) and by comparing the computed values of the characteristic impedance with those calculated from the well-known analytical expression [18].

Table 5 gives the capacitance matrix of a two-coductor boxed strip line computed using the finite difference method and the BEM. In the first case, a mesh size of $100 \times 20$ is used and the corresponding system of linear equations is solved using a successive over relaxation technique. With the BEM, however, a total of 100 elements on inner and outer conductors have been found sufficient to give results of comparable accuracy. Such a relatively small grid size can be easily handled on a moderate computer system using, for instance, a simple Gaussian elimination aigorithm.

\section{2- Multiconductor Lines Between Farallel Ground Planes}

This type of transmission lines is an example of potential problems where the boundaries involved extend to infinity. In boundary elements, as well as in tinite elements analyses, these infinite boundaries have been dealt with in various ways: 
simple truncation, use of infinite elements, or by combining numerical methods with appropriate analytical ones. Simple truncation, by introducing end walls at far enough distance, teduces the problem to one of the rype considered in the previous subsection but eventualiy increases the mesh size. Aiso, it has been found that the results depend on whether Dirichlet's or Neumann's condition is imposed on the artificial end wall [19]. On the other hand, the use of an element shape function extending to infinity and including an exponential decay does not involve much increase of the number of elements, but the choice of the rate of exponential decay is somewhat arbitrary and may affect the resuit [20].

The third approach, the one adopted here, consists in the following. As shown in the Ligures accompanying Tables 5 and 6, the entire domain between the ground planes is divided into two parts: an interior region I enclosing the conductors and bounded by the rectangular contour $B_{\text {, }}$, and an outer region il extending to infinity. Inside this latter homogeneous region the potential is represented by

$$
\begin{aligned}
& u=\Sigma A_{m} \exp (-m \pi|x| / L) \sin (m y / L) \\
& q=\Sigma A_{m} m \mid L \exp (-m \pi|x| L) \sin (m y / L)
\end{aligned}
$$

In the interior domain, the BEM is applied with the variation of the functions along the boundary elements assumed as linear. This means that

$$
\begin{aligned}
& u(y)=\Sigma u_{n} \phi_{n}(y) \\
& q(y)=\Sigma q_{n} \phi_{n}(y)
\end{aligned}
$$

where $\phi_{n}$ is a triangular pulse function with a unit peak at node $n$ and zeros at nodes $n+1$ and $n-1$, respectively. The BEM equarions are then formed in the usual way. However, since neither the potential nor its normal derivative is specitied along the interface $A B$ between regions $I$ and II. the number of unknowns would exceed the number of BEM equations unless further enough relations between the variables are provided. This is done by 'matching' the solutions in the exterior and interior domains along $\mathrm{AB}$.

One method is to set the porentials and their normal derivatives at the nodal points (i.e. the set of $u_{n}$ and $q_{n}$ values in equations (15)) equal to the corresponding values obtained from equations (14). Although this process ensures the coincidence of the exterior and interior-solutions at the nodal points, the two solutions may vary considerably over the whole element unless the element lentgth is too smali, which means an excessively large number of nodes and an increased memory requirement. The difference, or mismatch, berween the two solutions results in a discontunuity in potential across the element and is therefore physically equivalent to the introduction at the interface of a double layer which may result in an unacceptable error in potential and change distributions. It is therefore necessary that, for a given number of nodes, the difference between the interior and exterior soiutions along the common interface be kept a minimum, for instance, in the feast square sense. This can be effected by minimizing the error function

$$
F=\int\left(u_{e}-u_{i}\right)^{2}, w\left(q_{e}-q_{i}\right)^{2} d c
$$

where $w$ is some weighting function and the integration is along the interface AB. Substituting from equations (14) and (15) into (16) and equating the partial derivatives with respect to the nodal values $\left[u_{n}\right]$ and $\left[q_{n}\right]$ to zero, we get after eliminating the expansion coefficients $\left[A_{n}\right.$ ]

$$
\left(a_{1}, q_{2}, \ldots, c_{11}\right)^{\top}=c\left(v_{1}, v_{2}, \ldots, q_{n}\right)
$$

where $\mathrm{C}$ a square matrix of order $\mathrm{n}$. 


\section{E. 6 H. A. Elmikati}

The linear relation (17) between the nodal values on the interface $A B$ and the vanishing: of the potential on the upper and lower ground planes provide a sufficient set ut boundary conditions for solving the BEM equations in the interior region II.

For the purpose of comparison, we have applied the above approach to compute the rapacitance matrix for the structure shown with Table 5, but with the end walls removed. The results arc given in the same table together with the values obtaned by Karnmler using a Green's function integral equation technique [11]. The IBEM gives slighgtly higher values, presumably because we have assigned a very small (bur non-zero) thickncss to the strips. This has been found computionally more convenient as it allows the use of the routines developed for the boxed line with slight modifications and svoids troubles caused by singularities at the ends of all infitismally thin strip (although in principle these singularities can be dealt with in the manner discussed beluw).

Table 6 presents another example taken from reference [13] which uses aGreen's fuction integral equation approuch similar to the one developed earlier by Kammler [11\%. The same example has been solved in a recent work using a moment. method [15]. The BEM results are closer to the results of refrence [13] than to those of the momen- method in which the upper ground plane had to be truneater at a finite width.

finally, we present in fig. 2 an example of an infinitesmally thin microstrip over a dielectric substrate of linite width; a practical case whose analysis has recesved much less attention than the infinite-width substrate [21]. The BEM analiws of this problem is carricd out in the manner outlined above with the integration path taken as shown in lig. 2. The singularity due to the sudden change in boumlary condition at the edge of the microstrip has been treated by refining the mesh near this edge and by using a shape interpolation function proportional to the -yuare root of the distance Irom the edge, so as to satisfy the edge condition. This treatment of singularities in BEM is discussed in detail in reference [22]. Examination of the results presented in Fig. 2 shows that, unlike the narrow substrate, the IIEM results for the relatively wide substrate do not differ appreciably from the corresponding values for a microstrip over an infinite substrate reported in earlict work $[3,8]$. The present method can therefore be used for the anaiysis of the winite-width case, provided the substrate is truncated at an appropriate width.

\section{$\checkmark$ CONCLUSION}

Beside its simplicity and memory-economic character, the boundary element metiwd is capable of treating a ivide class of transverse electromagnetic transmission lines with an accuracy comparable with other numerical and analytical techniques. Problutns of shielded strip and microstrip lines involving infinite boundaries can be treated by combining BEM analysis with an appropriate expansion of the potential in the conductor free region 1 ithout resort to infinite elements, the accuracy of which may depend on arbitrary thosen parameter. in principle, the present techniques can be applied to problems involving multilayered cielectric media. Assessment of the method in such applications and a comparison with other techniques from the pwint of view of accuracy and memory requirements will be considered in a future' study.

\section{AC:KNOWLEDGMENT}

This work was carried $\mathrm{wu}$ in the Department of Electronic and Electrical Engukering University Collegr L.ndon. The author would like to thank Prof. J. 
B. Davies for providing support and for many useful discussions.

\section{REFERENCES}

[1] H. J, Ribiec, " An accurate determination of the characteristic impedance of the coaxial system consisting of a square concentric with a rircle. " IEEE Trans; Microwave Theory Techs, Vol. MTT-23, No, 8, pp, 714-715. Aug. 1975.

[ 2] H. A. Wheeler, "Transmission line properties of a round wire in a polyeonat shield, "IEEE Trans. Mierowave Theory Teche, Vol. MTT-27, pp+ 717-721. Aug. 1979,

[ 3 ] K. K. Joshi, 3. S. Rao, and B. N. Das, " Analysis of inhamogenously filled stripline and microstrip tine, "IEE Proc, Yol. 127, pt. No, 1. pp. $11-14_{1}$ Feb. 1980.

[4] Wergan Lin," A critical study of the coaxial transmission line utilizing conductors of both circular and square cross sectlon, "IEEE Trans. Microwave Theory Tech, Vol. MTT-30, No. 11, pp, 1981-1988, Nov. 1982.

[ 5] Weigan Lin, "Polyganal coaxial line with round center conductor, " IEEF Trans. Microwave Theory Tech-, Vol. MTT-33, No, 6, pp. 545-550, June 1985.

[6] K. V. Seshagiri and B. N. Das, "Impedance of an eltiptic conductor arbitrarily located between ground planes filled with two dielectric media, "IEEE Trans. Wicrowave Theory Tech-, Vol, MTT-33, No, 6, pp. 550-554, June 1985.

[7] E. Yamashita, "Variational method for the analysis of microstrip-like transmissinn lines, " IEEE Trans, Microwave Theory Tech., Vol, MTT-16, No, 8, Pp. 529-535, Aug. 1968,

(8) R. Mittra and T. itoh, "Charge and potential distributions in shielded steip lines, " IEEE Trans. Microwave Theoty Tech., Vol. MTT.j8, No. 3, pp. 149. 156, Dlarch 1970.

[9] M. Y. Schneider, " Computation of impedance and attenuation of TEMlines by finite difference methods, "IEEE Microwave Theory Tech.. Vol. MTT-13, No. 6, pp. 793-800, Nov. 1965.

[ ID] R. L. Brooke and I. E. Cruž, "Current distribution and impedance of lossiess conductor system, " IEEE Trans. Microwave Theory Tech., Vol. MTT. 15, No. 6, DP. 358-363, June 1976 .

[11] D. W. Kammier, "Calculation of characteristic admittanees and coupling coefficients for strip transmussion lines, "IEEE Trans. Microwave Theory Tech. Vol.MTT.16, No. 11, pp. 925-937, Nov. 1968.

[12] P. C. Chestnut, "On determining the capacitance of shieided multiconductor transmission lines, " IEEE Trans. Microwave Theory Tech., Vol. MTT-17. No. 10, pp. 734-745, Oct. 1969.

[13] W. T. Weeks, " Calculation of the coefficients of capacitance of multiconductor transmission lines in the presence of a deelectric interface, " IEEE Trans. Microwave Theory Tech., Vol. MTT-18, No. I, Pp. 35-43, Jan. 1970.

[14] w. T. Weeks, " Multiconductor transmision-line theory in the TEM approximation, " $18 \mathrm{M} 3$, RES., pp. 604-61 i, Nov. 1972. 
[15] C. Wei, R. F. Harrington, J. R. Mautz, and T. K. Sarkar, "Multiconductor transmission lines in multilayered dielectric media, "IEEE Trans. Microwave Theory Tech., Vol. MTT-32, No. 4, pp. 439-449, April 1984.

[16] C. A. Brebbia and S. Walker, Boundary Element Techniques in Engineering. Newnes-Butterworth, 1980.

[ 17] R. E. Collin, Field Theory of Guided Waves. New York, Mc-Graw Hill. 1963.

[ 18] S. Ramo, J. Whinnery, and T. Van Duzer, Fields and Waves in communication Electronics. John Wiley, New York 1965.

[ 19] M. Ikeuchi, " Boundary element analysis of shieided microstrip lines with dielectric layers, "Trans. IECE Japan (Section E), Vol. E-67, No. 11, pp. 585-590, Nov. 1984.

[ 20 ] P. Bettess, "Infinite elements, "Int. ]. Num. Meth. Engng., Vol. 11, pp. 53-64. 1977.

[21] C. E. Smith and R. Chang, "Microstrip transmission line with finite-width dielectric, "IEEE Trans. Microwave Theory Tech., Voi. MTT-28, No. 2 , pp. $70-94$, Feb. 1980.

[22] S. G. Lera, E. Paris, and E. Alarcon, "Treatment of singularities in 2-D domains using BIEM, "Appl. Math. Modelling, Vol, 6, pp. 111-118, April 1982. 


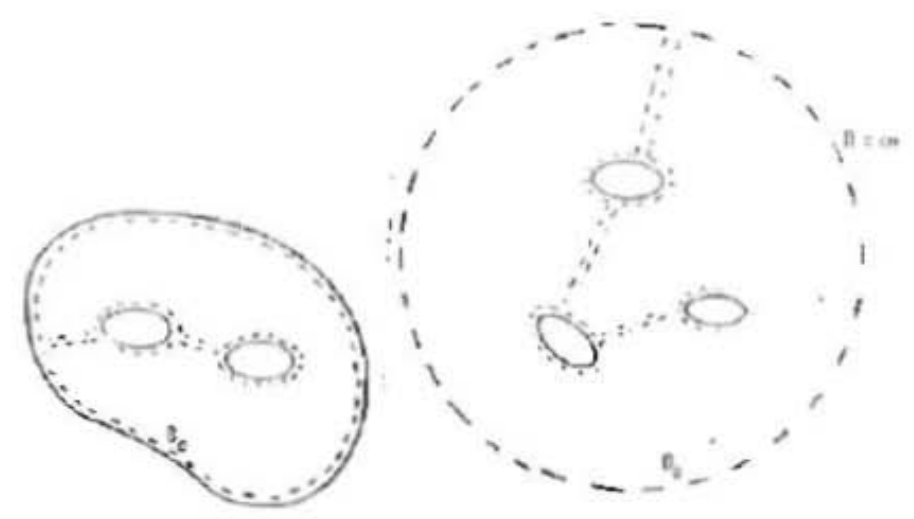

Fig. IShielded and open-wire TEM transmission lines. The dashed line is the BEM path of integration.

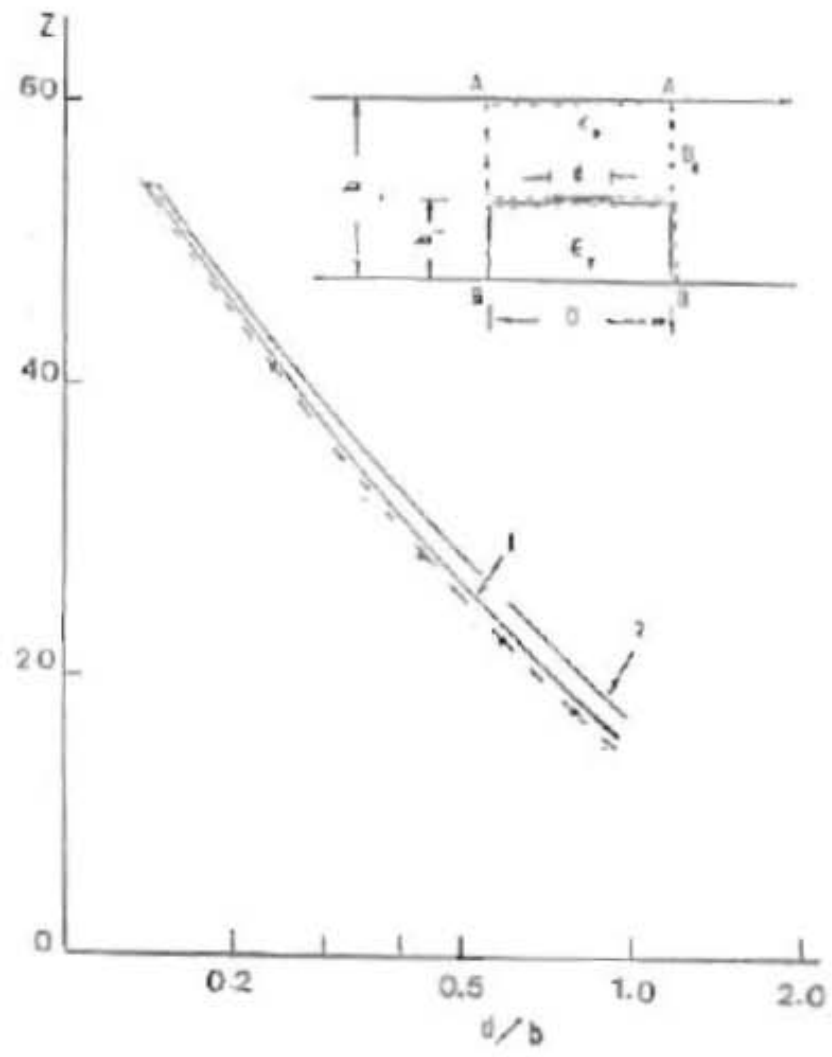

Fig. 2 A microstrip line problem. $=9.9, \mathrm{~b} / \mathrm{b}-1 / 6$. Curves 1 and 2 are BEM values for $\mathrm{d} / \mathrm{D}=0.2$ and 0.8 , respectively. The dois and the dashed line are values for an intinitc-width substrate (refrences $[3,8]$. 


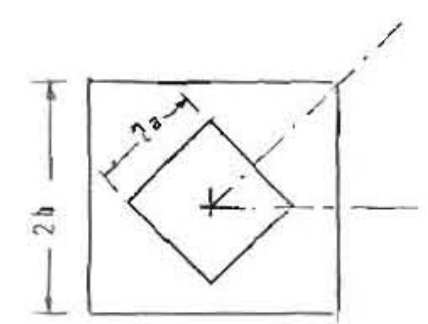

\begin{tabular}{ccc}
\hline$a / b$ & $z_{0}($ exact $)$ & $z_{0}(B E M)$ \\
\hline 0.638732 & 20.0 & 18.519 \\
0.547498 & 30.0 & 29.244 \\
0.464000 & 40.0 & 39.853 \\
0.388874 & 50.0 & 50.709 \\
0.323103 & 60.0 & 61.971 \\
0.232320 & 80.0 & 81.346 \\
0.168230 & 100.0 & 101.226 \\
0.121257 & 120.0 & 120.864 \\
\hline
\end{tabular}

Table 1. Exact (reference [4]) and BEM characteristic impedance of a coaxial dine of square inner and outer conductors.

Number of boundary elements $\mathrm{N}=60$.

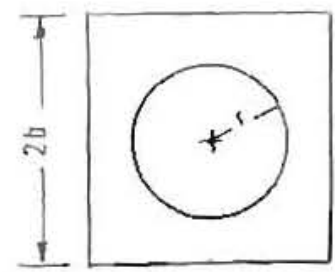

\begin{tabular}{llll}
\hline Ib & $z_{\min }$ & $z_{\max }$ & $z(\mathrm{BEM})$ \\
\hline 0.239085 & 90.315 & 90.372 & 0.401 \\
0.399780 & 69.199 & 69.319 & 9.291 \\
0.445150 & 52.840 & 53.217 & 3.129 \\
0.557536 & 39.139 & 39.999 & 9.569 \\
0.676602 & 27.031 & 28.912 & 7.970 \\
0. & 15.878 & 19.563 & 7.592 \\
0.933520 & 05.238 & 11.981 & 7.650 \\
\hline
\end{tabular}

Table 2. Lower $\left(z_{\text {min }}\right)$ and upper $\left(z_{\text {max }}\right)$ limits (reference [4]) and boundary element value of the characteristic impedance (ohms) of a coaxial line of square outer conductor and circular inner conductor. $N=60$. 

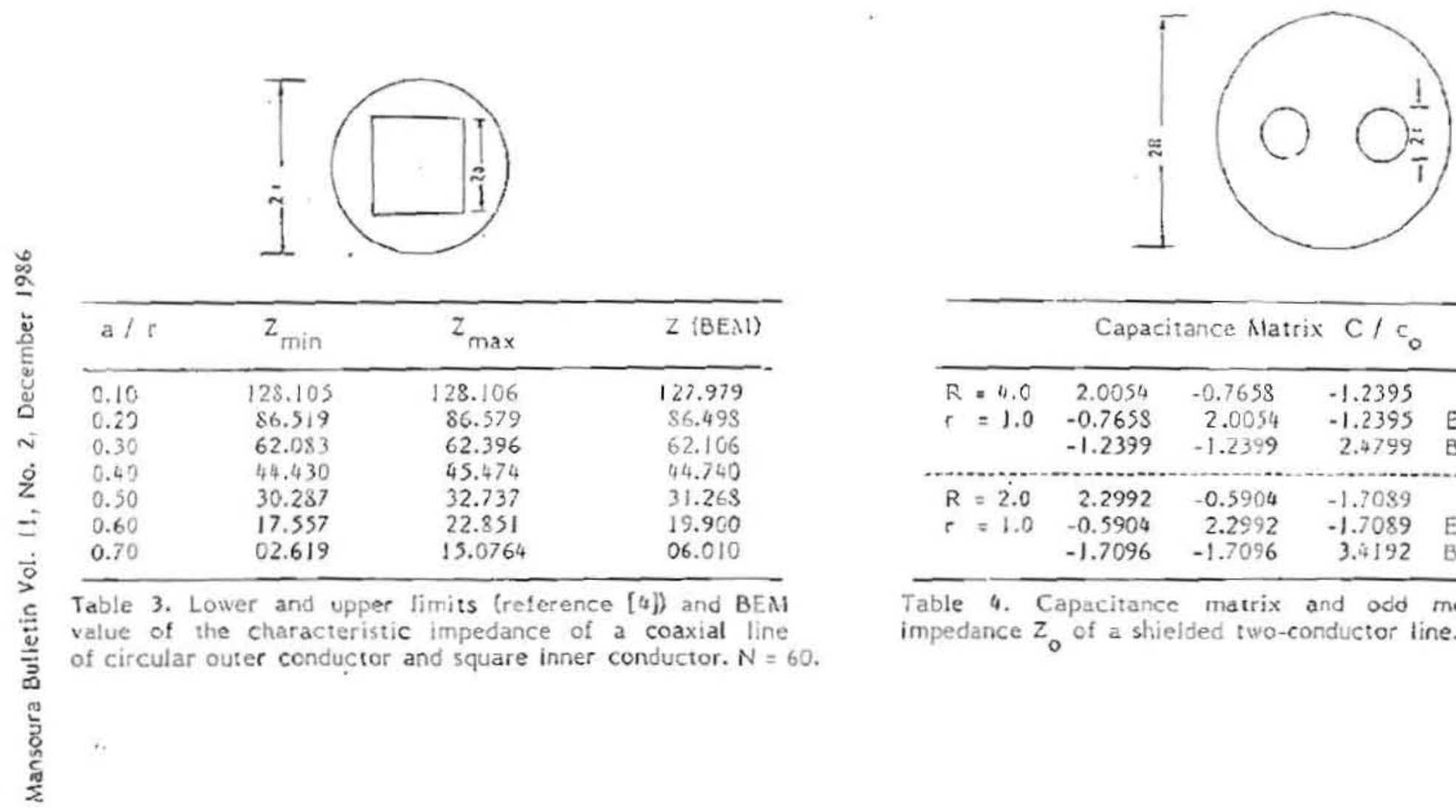

\begin{tabular}{|c|c|c|c|c|}
\hline & Capac & tance Nlatrix & $\mathrm{C} / \mathrm{c}_{0}$ & $z_{0}^{*}$ (ohms) \\
\hline $\begin{array}{l}R=4.0 \\
r=1.0\end{array}$ & $\begin{array}{r}2.0054 \\
-0.7659 \\
-1.2399\end{array}$ & $\begin{array}{r}-0.7658 \\
2.0054 \\
-1.2399\end{array}$ & $\begin{array}{r}-1.2395 \\
-1.2395 \\
2.4799\end{array}$ & $\begin{array}{l}\text { Exact value } 271.42 \\
\text { BEM value } 271 . \$ 9\end{array}$ \\
\hline $\begin{array}{l}R=2.0 \\
r=1.0\end{array}$ & $\begin{array}{r}2.2992 \\
-0.5904 \\
-1.7096\end{array}$ & $\begin{array}{r}-0.5904 \\
2.2992 \\
-1.7096\end{array}$ & $\begin{array}{r}-1.7089 \\
-1.7089 \\
3.4192\end{array}$ & $\begin{array}{l}\text { Exact value } 260.38 \\
\text { BEAt value } 265.75\end{array}$ \\
\hline
\end{tabular}

Table 4. Capacitance matrix and odd mode characteristic impedance $Z_{0}$ of a shielded two-conductor tine. $N=60$. 

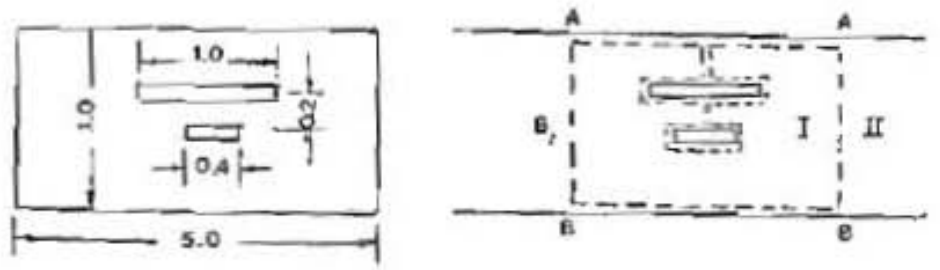

\begin{tabular}{|c|c|c|c|c|c|}
\hline \multicolumn{6}{|c|}{ Capacitance Coefficients $\mathrm{C} / \mathrm{C}_{\mathrm{o}}$} \\
\hline & & $\sigma_{11}$ & $c_{12}$ & $c_{21}$ & $c_{22}$ \\
\hline $\begin{array}{l}\text { Boxed- } \\
\text { Line }\end{array}$ & $\begin{array}{l}\text { Finite Dilf. } \\
\text { BEM }\end{array}$ & $\begin{array}{l}8.1688 \\
8.1568\end{array}$ & $\begin{array}{l}-3.2416 \\
-3.1686\end{array}$ & $\begin{array}{l}-3.2399 \\
-3.1750\end{array}$ & $\begin{array}{l}5.0721 \\
4.9410\end{array}$ \\
\hline $\begin{array}{l}\text { Open- } \\
\text { ended Line }\end{array}$ & $\begin{array}{l}\text { Integral Eqn. } \\
\text { BEM }\end{array}$ & $\begin{array}{l}7.9039 \\
7.9636\end{array}$ & $\begin{array}{l}-3.0640 \\
-3.1024\end{array}$ & $\begin{array}{l}-3.0640 \\
-3.0978\end{array}$ & $\begin{array}{l}4.8074 \\
4.8465\end{array}$ \\
\hline
\end{tabular}

Table 5. Elements of the capacitance matrix of a two-conductor shielded strip line computed by the BEM, finite differences,and integral equation method of reference $[11] . \mathrm{N}=100$.

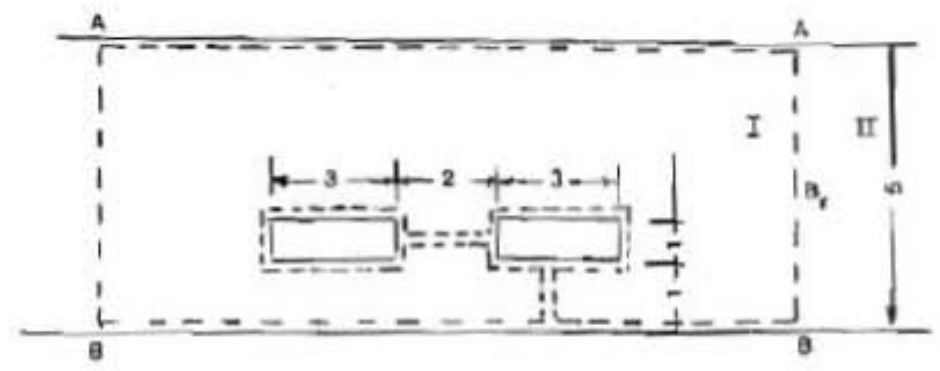

\begin{tabular}{llll} 
& Integral Eqn. [ 13 ] & Moment Method [ I5 ] & BEM \\
\hline$C_{11}$ & 63.07 & 62.64 & 64.21 \\
$C_{12}$ & -05.866 & -05.724 & -05.923 \\
$C_{21}$ & -05.866 & -05.724 & -05.923 \\
$C_{22}$ & 63.07 & 62.64 & 64.21
\end{tabular}

Table 6. Elements of the capecitince matrix $\left(\times 10^{12} \mathrm{~F} / \mathrm{m}\right)$ for a shielded two-conductor line. $\mathrm{N}=100$. 